\title{
A novel plant gene essential for meiosis is related to the human CtIP and the yeast COM1/SAE2 gene
}

\author{
Clemens Uanschou ${ }^{1,5}$, Tanja Siwiec ${ }^{1,5}$, \\ Andrea Pedrosa-Harand ${ }^{1,5,6}$, Claudia \\ Kerzendorfer ${ }^{1,2}$, Eugenio Sanchez-Moran³, \\ Maria Novatchkova4, Svetlana Akimcheva', \\ Alexander Woglar ${ }^{1}$, Franz Klein ${ }^{1}$ and \\ Peter Schlögelhofer ${ }^{1, *}$
}

${ }^{1}$ Department of Chromosome Biology, Max F Perutz Laboratories,
University of Vienna, Vienna, Austria, ${ }^{2}$ Gregor Mendel Institute of
Molecular Plant Biology, Austrian Academy of Sciences, Vienna,
Austria, ${ }^{3}$ School of Bioscience, University of Birmingham, Birmingham,
UK and ${ }^{4}$ Research Institute of Molecular Pathology, Vienna, Austria

Obligatory homologous recombination (HR) is required for chiasma formation and chromosome segregation in meiosis I. Meiotic HR is initiated by DNA double-strand breaks (DSBs), generated by Spo11, a homologue of the archaebacterial topoisomerase subunit Top6A. In Saccharomyces cerevisiae, Rad50, Mre11 and Com1/Sae2 are essential to process an intermediate of the cleavage reaction consisting of Spo11 covalently linked to the $5^{\prime}$ termini of DNA. While Rad50 and Mre11 also confer genome stability to vegetative cells and are well conserved in evolution, Com1/Sae2 was believed to be fungal-specific. Here, we identify COM1/SAE2 homologues in all eukaryotic kingdoms. Arabidopsis thaliana Com1/Sae2 mutants are sterile, accumulate AtSPO11-1 during meiotic prophase and fail to form AtRAd51 foci despite the presence of unrepaired DSBs. Furthermore, DNA fragmentation in AtCom1 is suppressed by eliminating AtSPO11-1. In addition, AtCOM1 is specifically required for mitomycin $\mathrm{C}$ resistance. Interestingly, we identified CtIP, an essential protein interacting with the DNA repair machinery, as the mammalian homologue of Com1/Sae2, with important implications for the molecular role of CtIP.

The EMBO Journal (2007) 26, 5061-5070. doi:10.1038/

sj.emboj.7601913; Published online 15 November 2007

Subject Categories: genome stability \& dynamics

Keywords: Arabidopsis; CtIP; DNA repair; meiosis; SAE2

\section{Introduction}

The process of meiosis includes two successive nuclear divisions that follow a single round of DNA replication. During the reductional division, called meiosis I, pairs of

\footnotetext{
${ }^{*}$ Corresponding author. Department of Chromosome Biology, Max F Perutz Laboratories, University of Vienna, Dr Bohr-Gasse 1, Vienna A-1030, Austria. Tel.: + 4314277 56240; Fax: + 4314277 9562; E-mail: peter.schloegelhofer@univie.ac.at

${ }^{5}$ These authors contributed equally to this work

${ }^{6}$ Present address: Department of Botany, Federal University of Pernambuco, 50670-420, Recife, Brazil
}

Received: 18 June 2007; accepted: 17 October 2007; published online: 15 November 2007 replicated homologous chromosomes segregate, while during meiosis II, sister chromatids are separated in an equationallike division. During prophase of meiosis I, reciprocal recombination between homologous chromosomes occurs before they are segregated at meiosis I. The process of homologous recombination (HR) contributes to the genetic diversity of the meiotic products and is a prerequisite for the correct segregation of homologous chromosomes (Zickler and Kleckner, 1999; Page and Hawley, 2003).

Meiotic recombination is initiated by DNA double-strand breaks (DSBs), generated by Spo11, a homologue of the archaebacterial topoisomerase subunit Top6A (Bergerat et al, 1997; Keeney et al, 1997). Arabidopsis thaliana has three SPO11-like genes, while all other analysed species possess only one such gene (Hartung and Puchta, 2000, 2001). Arabidopsis Atspo11-1 (Grelon et al, 2001) and Atspo11-2 mutants (Stacey et al, 2006) exhibit defects in pairing and recombination during meiosis, while Atspo11-3 is not involved in meiosis (Yin et al, 2002). In budding yeast, the help of at least nine proteins (Smith and Nicolas, 1998; Keeney, 2001; Prieler et al, 2005) is required for Spo11mediated catalytic cleavage. Further processing of DSBs requires some of the same and several additional proteins, among them Com1/Sae2 (Keeney and Kleckner, 1995; McKee and Kleckner, 1997; Prinz et al, 1997; Cartagena-Lirola et al, 2006). Spo11 is found covalently linked to the $5^{\prime}$ termini of DNA in an intermediate of the DNA cleavage reaction. The release of Spo11 from DNA is mediated by ssDNA nick formation next to the DSB site, thereby liberating the Spo11 protein attached to a few nucleotides. In budding yeast, this release requires Rad50, Mre11 and Com1/Sae2. Mutations in these genes cause accumulation of Spo11 on DNA ends (Keeney and Kleckner, 1995; Neale et al, 2005; Prieler et al, 2005). Following DNA cleavage and Spo11 removal, a $5^{\prime}-3^{\prime}$ exonucleolytic activity, mediated by a nuclease not yet identified, generates 3 ' single-stranded DNA tails. These tails are believed to serve as probes for identifying homologous partner chromosomes, and to initiate D-loop formation and single-end invasion followed by second-end capture (Paques and Haber, 1999; Hunter and Kleckner, 2001; Neale and Keeney, 2006).

Homologues of RAD50, MRE11 and their complex partner $X R S 2 / N B S 1$ have been identified in higher eukaryotes, while homologues of Com1/Sae2 have remained elusive. Yeast twohybrid assays have demonstrated that the Arabidopsis proteins AtRAD50 and AtMRE11 physically interact and that the rice OsMRE11 protein interacts with OsNBS1 (Bhatt et al, 2001; Daoudal-Cotterell et al, 2002; Akutsu et al, 2007). A mutation in AtRAD50 leads to meiotic defects, sterility and sensitivity to methyl-methano-sulphonate (Gallego et al, 2001; Bleuyard et al, 2004). In vertebrates, MRE11 is an essential gene with roles in both somatic and meiotic cells (D'Amours and Jackson, 2002), while in A. thaliana, MRE11 is not essential. Depending on the MRE11 mutant allele, plants are either sterile due to perturbed meiosis and exhibit 
severe developmental abnormalities or they only display enhanced sensitivity to genotoxic agents (Bundock and Hooykaas, 2002; Puizina et al, 2004). In contrast to yeast, MRE11 and RAD50 are not essential for the formation of meiotic DSBs in A. thaliana, but only in the ensuing processing and repair (Bleuyard et al, 2004; Puizina et al, 2004).

Yeast's COM1/SAE2 was detected independently in three genetic screens. Two screens were designed to isolate meiotic mutants, defective after the initiation of Spo11-induced DSBs, but before resolution of recombination intermediates (McKee and Kleckner, 1997; Prinz et al, 1997). The phenotypes of the isolated com1/sae2-null mutations are similar to those conferred by the previously identified non-null mutations of RAD50 (rad50S) and MRE11 (mre11S) (Alani et al, 1990; Keeney et al, 1997; Nairz and Klein, 1997; Tsubouchi and Ogawa, 1998). These mutations completely block resection and turnover of meiosis-specific DSBs. The third screen aimed to identify mutants with low fidelity DSB repair during vegetative growth (Rattray et al, 2001). In mitotic cells, Com1/Sae2 (together with proteins of the Mre11 complex) is essential for the repair of hairpin-capped DSBs, for holding DNA ends in close proximity after DSB formation and for preventing chromosome rearrangements (Lobachev et al, 2002; Clerici et al, 2005). This explains how Com1/Sae2 could confer resistance to genotoxic treatments (Birrell et al, 2002; Deng et al, 2005). Furthermore, detailed analysis has shown that Com1/Sae2 is phosphorylated by the protein kinases Mec1 and Tel1 (yeast homologues of ATR and ATM, respectively) and that it interferes with the DNA replication and damage checkpoints during mitosis and meiosis (Baroni et al, 2004; Cartagena-Lirola et al, 2006; Clerici et al, 2006). Com1/Sae2 is among the first proteins to be found at somatic DSB sites, after Mre11 and Tel1, but before Rad52. Com1/ Sae2 and Mre11 do not rely on each other with respect to their initial localisation to DSBs, but mutually depend on each other for their timely disappearance from DSBs (Lisby et al, 2004; Clerici et al, 2006).

The analysis of the A. thaliana AtCOM1 gene represents the first characterisation of COM1/SAE2 in a higher eukaryote. Our data demonstrate that a COM1/SAE2 homologue exists in various higher eukaryotes and that it is essential for female and male meiosis in A. thaliana. We show that AtCOM1 acts downstream of AtSPO11-1 and upstream of AtDMC1 during meiosis. We provide evidence that AtCOM1 is needed for regular turnover of AtSPO11-1 and processing of meiotic DSBs. Furthermore, we show that AtCOM1 is essential for a specific DNA repair process in somatic cells. Importantly, our findings correlate yeast's Com1/Sae2 protein with the mammalian DNA repair-related protein CtIP, whose significance in meiosis has not yet been elucidated.

\section{Results}

\section{Identification of COM1/SAE2 homologues in higher eukaryotes}

To identify homologues of the yeast COM1/SAE2 gene, we first collected a set of fungal Com1/Sae2 homologues by applying PSI-BLAST and reciprocal proteome BLAST searches (with low-complexity filtering, E-value cut-off 0.001) (Altschul et al, 1997) starting with the full-length Saccharomyces cerevisiae Com1/Sae2 protein sequence. Alignment of 20 different fungal Com1/Sae2 homologues revealed conservation of its C-terminal half (conservation plot (Rice et al, 2000)), corresponding to amino acids 190-345 of the yeast Com1/Sae2 protein. Consequently, reciprocal PSI-BLAST searches (against the NCBI non-redundant database (nr), version 12/2006; low-complexity filtered, inclusion cut-off 0.001) (Altschul et al, 1997; Marchler-Bauer et al, 2002) were carried out with the conserved region of all fungal relatives (Supplementary Figure S1A). This procedure yielded a coherent set of related sequences in a wide variety of eukaryotes including the Arabidopsis NP_850683 (At3g52115) (Supplementary Figure S1B) and the metazoan CtIP protein (Homo sapiens CtIP, Rbbp8; Fusco et al, 1998), shown in Figure 1A aligned by their common C-terminal homology. The Arabidopsis and the human proteins share $54 \%$ identity and $45 \%$ similarity in their conserved C-termini and their close relation are further illustrated by cluster analysis (Figure 1B; Frickey and Lupas, 2004). Arabidopsis At3g52115 was picked up in a screen as being upregulated after ionising irradiation and was called AtGR1 (A. thaliana gamma response gene 1) (Deveaux et al, 2000). Garcia et al (2003) demonstrated that ATM kinase is required for transcriptional induction of AtGR1. However, neither AtGR1's relation to COM1/SAE2, nor its importance for meiosis was noticed. We suggest calling the gene AtGR1/COM1 and hereafter refer to it as AtCOM1. The CtIP protein was also not recognised as a homologue of COM1/SAE2 despite a more detailed characterisation (see Discussion).

To underline the functional significance of the conserved COM1/SAE2 C-terminus, yeast $\Delta$ com 1 strains (Prinz et al, 1997) where transformed with plasmids either carrying wildtype or mutagenised versions of the COM1/SAE2 gene, with single, double or triple amino-acid exchanges of the most conserved residues, respectively. Whereas the cells which obtained a wild-type COM1/SAE2 gene showed $100 \%$ spore viability (as opposed to the untransformed $\Delta c o m 1$ strain which has $0.06 \%$ spore viability), the cells which obtained a mutagenised version showed strongly reduced spore viability: (1) R262,264A: 3.4\%; (2) S267A: 10.4\%; (3) P268A: 0.28\%; (4) GF270AA: $0.03 \%$; (5) FPS276AAA: 0.7\% (Figure 1A).

\section{Molecular characterisation of Atcom1}

To study the function of the AtCOM1 protein in plants, we obtained two T-DNA insertion mutant lines from the Salk Institute Genomic Analysis Laboratory T-DNA collection (Alonso et al, 2003) harbouring alleles Atcom1-1 and Atcom 1-2, respectively. We confirmed the position of the Atcom 1-1 and Atcom1-2 T-DNA insertions within the 2nd exon of the AtCOM1 gene by PCR, sequenced the insertion borders and determined the structure of Atcom1-1 by Southern analysis (Supplementary Figure S2A-C). Expression analysis by reverse transcription revealed the presence of AtCOM1 transcripts in the all wild-type tissues examined (buds, leaves, seedlings), and a strong induction in irradiated tissues. Only a truncated mRNA, corresponding to the region upstream of the T-DNA insertion sites, was detected, in the mutant plants (Supplementary Figure S2D). As both the Atcom1-1 and the Atcom 1-2 coding regions are interrupted by a stop codon (Supplementary Figure S2B), the mutant proteins are predicted to consist of the N-terminal 456 and 479 amino acids, respectively, both lacking the conserved C-terminus (the full-length protein has a predicted length of 588 amino acids). 
A

$\begin{array}{ll}\text { Sc_P46946 } & 204 \\ \text { Ag_AAS51872 } & 223 \\ \text { Y1_XP_502193 } & 280 \\ \text { An_XP_662716 } & 635 \\ \text { Gz_EAA69989 } & 545 \\ \text { Nc_XP_323685 } & 455 \\ \text { Cn_EAL19137 } & 383 \\ \text { Ch_XP_667705 } & 187 \\ \text { Dr_NP_001012518 } & 545 \\ \text { X1_AAH73395 } & 752 \\ \text { Hs_NP_976036 } & 793 \\ \text { Mm_AAH50849 } & 789 \\ \text { Dd_XP_635231 } & 366 \\ \text { At_NP_850683 } & 513 \\ \text { Os_BAD35559 } & 494 \\ \text { Dm_NP_649224 } & 406 \\ \text { Dp_EAL30480 } & 414 \\ \text { Consensus } & 90 \% \\ \text { Consensus } & 70 \% \\ \text { Consensus } & 50 \% \\ & \end{array}$

Quality

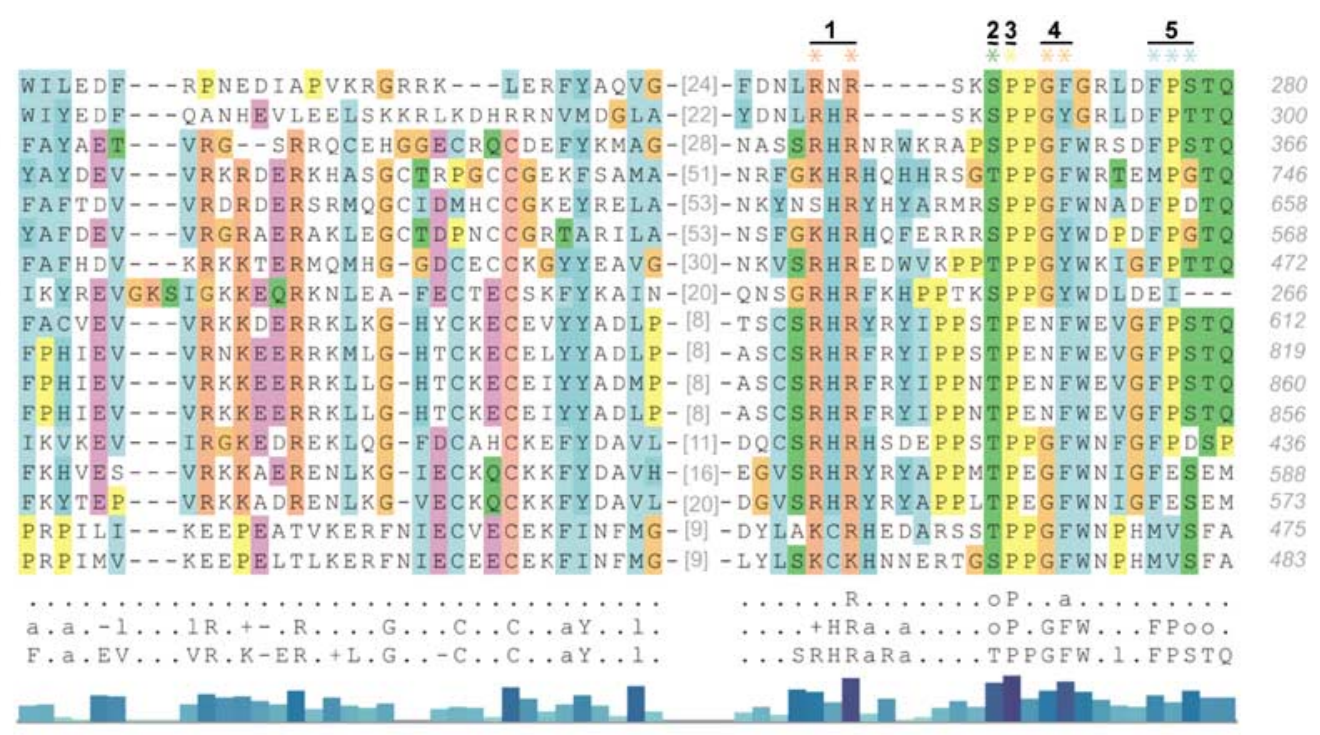

B
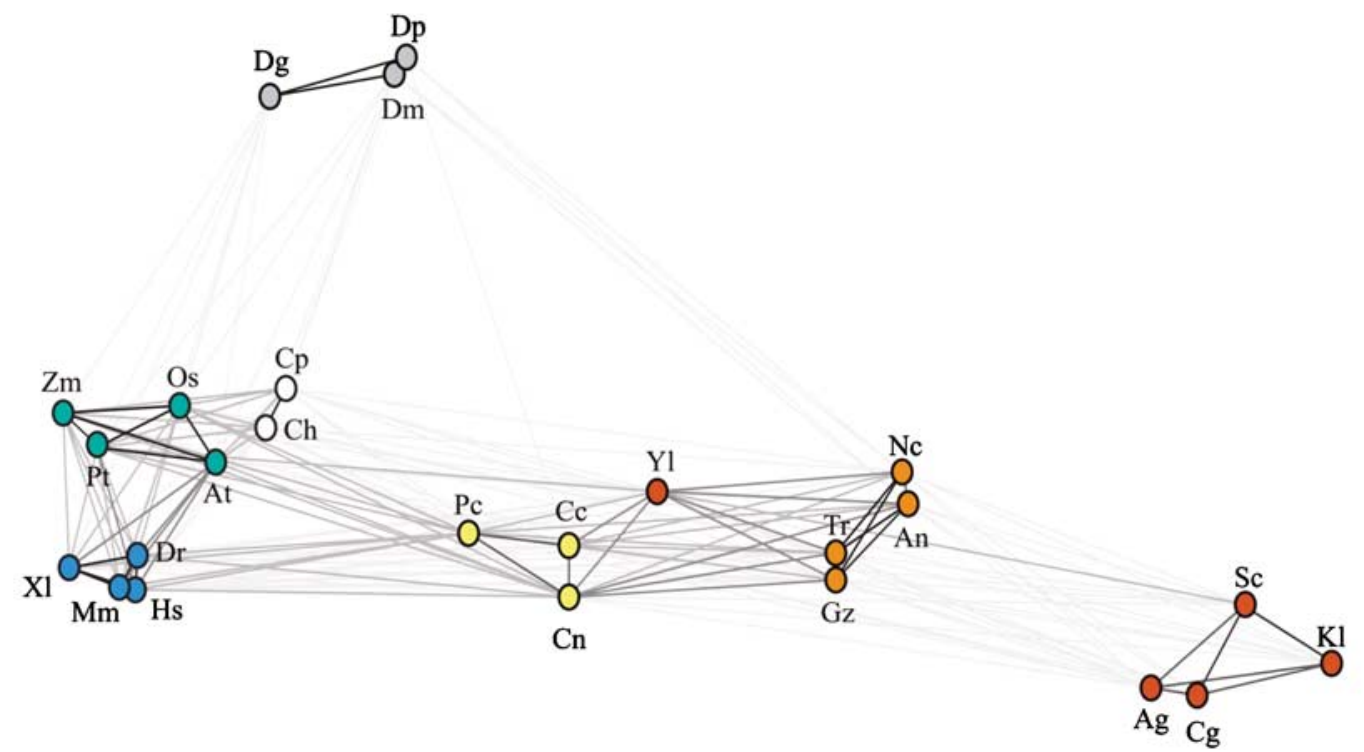

Figure 1 The Com1/Sae2 motif is conserved among eukaryotes. (A) Alignment of putative homologues of the S. cerevisiae Com1/Sae2 protein. The alignment (muscle (Edgar, 2004); clustal-like colouring) shows the C-terminal protein region of best conservation. Residue conservation above selected thresholds $(50,70,90 \%)$ is indicated below the alignment. Relative frequency was scored for individual amino acids as well as for groups of related amino acids: alcoholic (denoted by 'o' and including the amino acids S, T), aliphatic ('l' for I, L, V), aromatic ('a' for F, H, $\mathrm{W}, \mathrm{Y}$ ), positive ('+' ' for H, K, R), negative ('-' for D, E). Numbers and asterisks above the alignment refer to amino-acid exchanges introduced into the S. cerevisiae protein sequence. (B) Cluster analysis of COM1/SAE2 homologues. The analysis was performed with CLANS (Frickey and Lupas, 2004). Individual proteins are shown as vertices and are connected by lines reflecting pairwise sequence similarities from all-against-all BLAST searches. Attractive forces increasing with the sequence similarity between proteins lead to clustering of sequence related proteins. The strength of line shading increases with increasing sequence similarity. Abbreviations: (Ag) Ashbya gossypii, (An) Aspergillus nidulans, (At) Arabidopsis thaliana, (Cc) Coprinopsis cinerea, (Cg) Candida glabrata, (Ch) Cryptosporidium hominis, (Cn) Cryptococcus neoformans, (Cp) Cryptosporidium parvum, (Dd) Dictyostelium discoideum, (Dg) Drosophila grimshawi, (Dm) Drosophila melanogaster, (Dp) Drosophila pseudoobscura, (Dr) Danio rerio, (Gz) Gibberella zeae, (Hs) Homo sapiens, (Kl) Kluyveromyces lactis, (Mm) Mus musculus, (Nc) Neurospora crassa, (Os) Oryza sativa, (Pc) Phanerochaete chrysosporium, (Pt) Populus trichocarpa, (Sc) Saccharomyces cerevisiae, (Tc) Trichoderma reesei, (Xl) Xenopus laevis, (Yl) Yarrowia lipolytica, ( $\mathrm{Zm})$ Zea mays.

\section{Atcom1 mutant plants are sterile due to chromosome fragmentation during meiosis}

Plants homozygous for the Atcom 1 mutant alleles (Atcom1-1, Atcom 1-2) do not show growth aberrations during vegetative development. The mutants germinate and develop at the same time and rate as wild-type plants. Rosette leaves are normal in size, shape and number, and bolting is not delayed. Inflorescences look normal, but none of the siliques of Atcom 1 mutant plants elongate, whereas shoots of wildtype plants have long siliques. The short siliques of
Atcom 1-1 and Atcom1-2 mutant plants are completely devoid of seeds (Figure 2A). This and all the other observed phenotypes were completely reversed by introduction of a genomic wild-type copy of the gene (Figure 2A). To further characterise the fertility defect, male and female gametophyte development was monitored. Atcom 1 mutant plants develop anthers that do not contain viable pollen (Figure 2A) and only aberrant embryo sacs (Supplementary Figure S3A), implying that the sterility phenotype of Atcom 1 mutant plants is based on gametogenesis defects. This conclusion is sup- 
ported by the fact that Atcom 1-1 plants ( $n=8$ buds) could not be fertilised with wild-type pollen. Prior to gametogenesis, haploid cells have to be formed during the process of meiosis. A key step during meiosis is synapsis and recombination between homologous chromosomes. Cytological analysis of male meiosis fails to detect synapsed chromosomes in Atcom 1-1 plants during stages corresponding to zygotene and pachytene (Figure 3B, C, M, N). To identify homologous chromosomes, chromosome I (at an interstitial region) and chromosome II (at a subtelomeric region) were simultaneously labelled by fluorescent in situ hybridisation (FISH). Indeed, no close pairing of either homologous signal pair was observed in the mutant (Figure $3 \mathrm{M}, \mathrm{N}$ ), but close pairing was frequently seen in wild-type zygotene stages and always observed in wild-type pachytene stages (Figure 3B, C). This implies that no stable interactions between homologous chromosomes can be formed in homozygous Atcom 1-1. While five well-defined bivalents emerge from condensation during diakinesis (Figure 3E) and metaphase I in wild type (Figure 3F), chromosomes are always connected and entangled in the Atcom1-1 mutant during stages corresponding
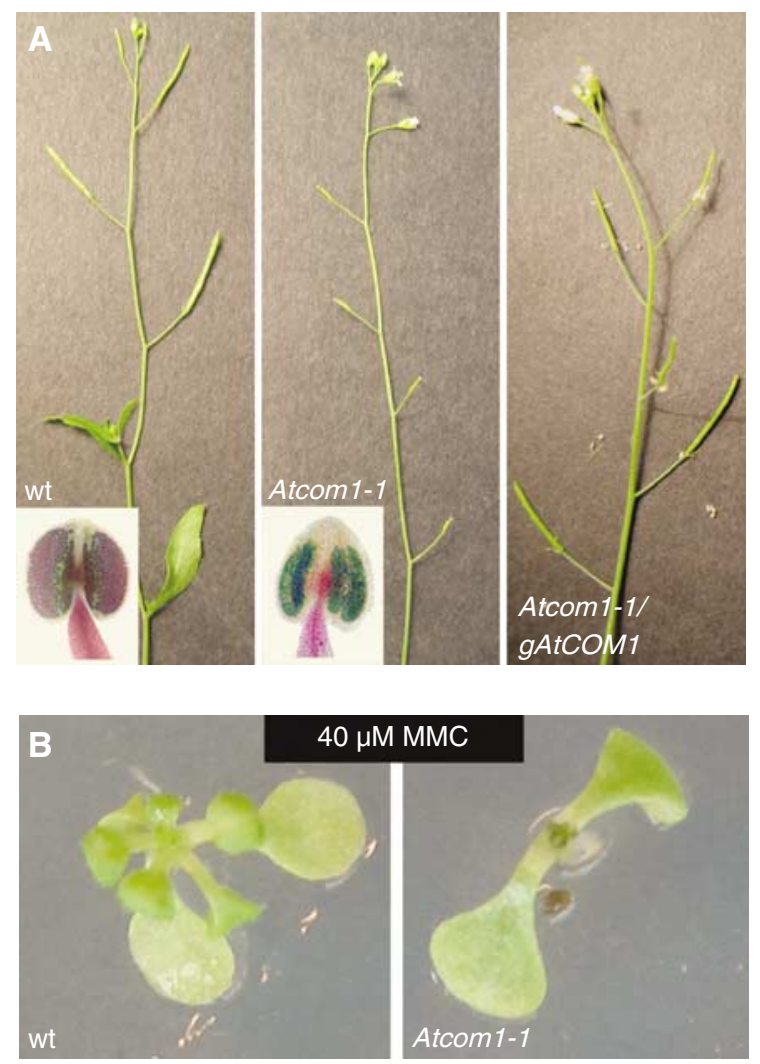

Figure 2 Phenotypes of the Atcom 1-1 mutant. (A) Atcom 1-1 mutants look like wild-type plants, but do not develop mature siliques. The left panel shows a stem with mature siliques of a wild-type (wt) plant. The middle panel shows the stem of an Atcom1-1 mutant plant of the same age, which failed to develop mature siliques. The right panel shows an Atcom1-1 mutant plant containing a fertilityrestoring genomic copy of AtCOM1. Inlays: anthers of wild-type (wt) and Atcom1-1 plants stained as described (Alexander, 1969). The purple-stained cytoplasm indicates viable pollen grains; green indicates empty pollen. No regular sized and viable purple pollen is present in Atcom 1 anthers. (B) Seedlings grown on media plates containing $40 \mu \mathrm{M}$ MMC. Wild-type (wt) plants develop true leaves. In contrast, Atcom1-1 mutant plants only expand their cotyledons but do not develop true leaves. to diakinesis and metaphase I (Figure 30, P). In anaphase I DNA fragments, typically telomeric fragments are left behind at the metaphase plate in Atcom 1-1 plants (Figure 3Q-S), indicating that meiotic DSBs remain unrepaired in the mutant. Figure 3R also shows a DNA bridge connecting two centromeres, which segregate to different poles. FISH analysis with a probe directed to the $180 \mathrm{bp}$ repeat region of centromeres shows that some of the bridges observed in Atcom1-1 mutants consist of centromeric DNA, indicating that bridges could either originate from separation of sister chromatids, persisting DNA catenation or from a nonhomologous repair mechanism (data not shown). DNA fragments and bridges are never observed in wild-type cells during anaphase or telophase I (Figure 3G, H). In addition to fragmentation, massive chromosome missegregation is revealed by the often asymmetric distribution of nuclear material in the first meiotic division in Atcom 1-1 cells (Figure 3). These are expected consequences of the earlier homologue pairing and DSB repair defects. Anaphase II figures were also observed, and all showed a high incidence of fragmentation and missegregation, usually culminating in the formation of more than four, poorly condensed, unequal masses of chromatin at telophase II (Figure 3U). Equivalent defects were observed during female meiosis in Atcom1-1 mutants (Supplementary Figure S3B). We conclude that the sterility of Atcom1-1 mutants is caused by aberrant meiosis and defective repair of meiotic DSBs.

\section{Chromosome fragmentation observed in Atcom1-1 mutants depends on SPO11-1 but not on the RecA-related DMC1 protein}

If fragmentation and chromosome bridges in Atcom 1-1 meiosis were solely caused by the inability to process meiotic DSBs, reduction of DSB formation by mutating the nuclease, AtSPO11-1 (Grelon et al, 2001), should strongly curb such aberrations (the Atspo11-1-1 mutation does not completely eliminate DSBs, either due to residual activity or due to its paralog AtSPO11-2 (Stacey et al, 2006)). Indeed, when we generated the homozygous Atcom1-1 Atspo11-1-1 double mutant, DNA fragmentation was undetectable in most cells (Figure 4), showing that the inability to repair meiotic DSBs is responsible for Atcom 1-1 chromosome aberrations.

Cells repair meiotic DSBs by using the homologue rather than the sister chromatid as a template, a phenomenon called interhomologue bias (Zickler and Kleckner, 1999). We, therefore, asked whether relaxing interhomologue bias might permit repair in Atcom1-1 meiocytes. DMC1, a meiosis-specific RecA recombinase, is specifically required for interhomologue interactions (Schwacha and Kleckner, 1997) and Atdmc1 mutants are thought to repair all DSBs by using the sister chromatid as a template (Couteau et al, 1999). However, chromosome fragmentation persists in the Atcom 1 Atdmc1 double mutant meiosis (Figure 4), suggesting that Atcom1 affects both intersister and interhomologue recombination alike. This result is expected, if AtCOM1 is not specific to the interhomologue repair pathway, but obligatory for meiotic repair as Com1/Sae2 is in yeast.

\section{AtCOM1 is essential for regular turnover of AtSPO11-1 and normal processing of DSBs}

A very early step in meiotic DSB repair after removal of Spo11 and formation of $3^{\prime}$ single-stranded ends is coating 

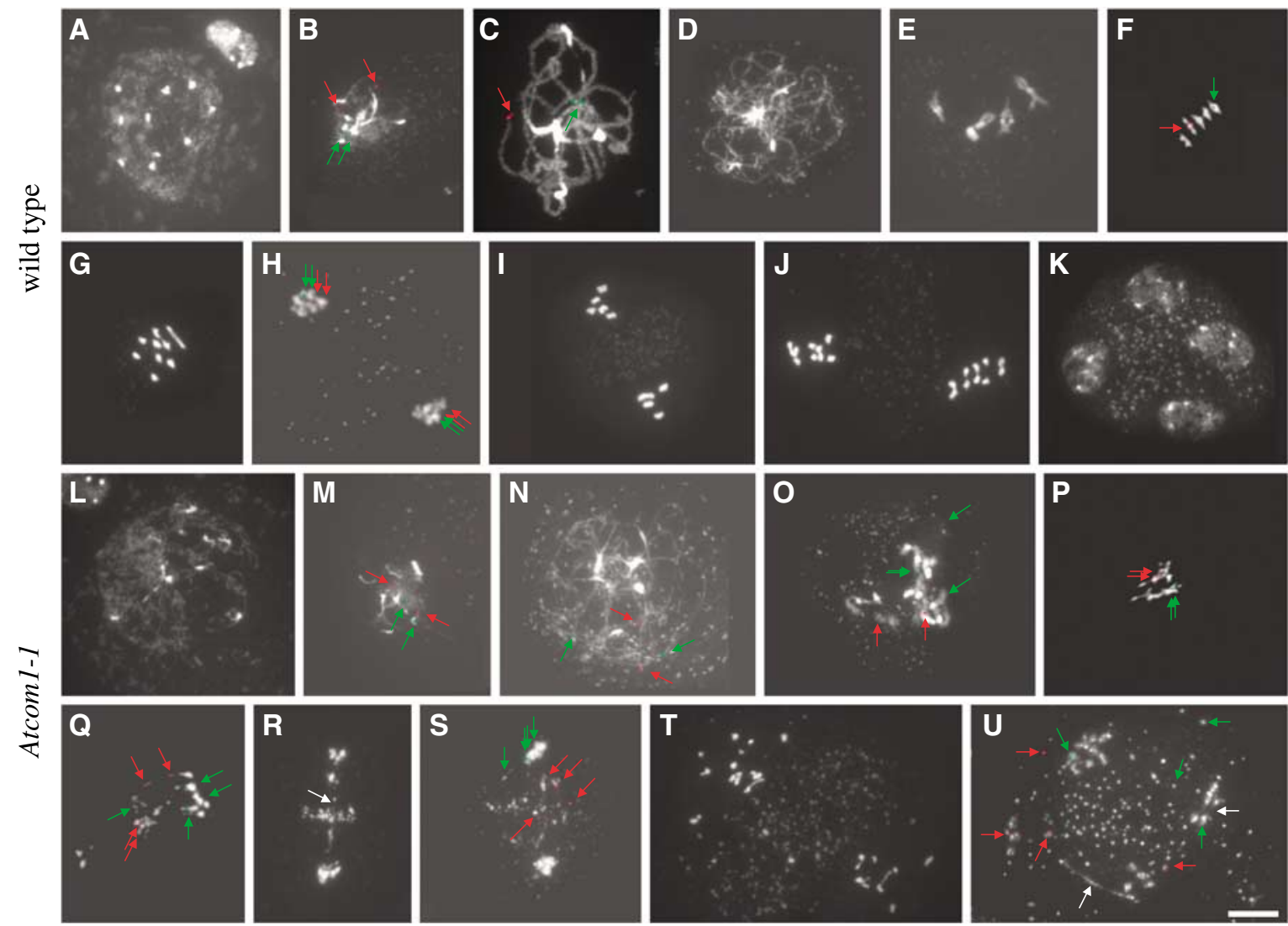

Figure 3 Meiosis in pollen mother cells of wild-type (A-K) and Atcom1-1 mutant (L-U) plants. Wild-type: (A) leptotene; (B) zygotene; (C) pachytene; (D) diplotene; (E) diakinesis; (F) metaphase I; (G) anaphase I; (H) prophase II; (I) metaphase II; (J) anaphase II; (K) telophase II. Meiosis in the severely disrupted Atcom1-1 mutant plants: (L) leptotene; (M) zygotene-like stage; (N) pachytene-like stage, without normal chromosome pairing; (O) diakinesis-like stage; (P) metaphase I-like stage with entangled chromosomes; $(\mathrm{Q}, \mathrm{R})$ progression of anaphase I with fragmentation of chromosomes; (S) prophase II-like stage; (T) anaphase II-like stage; (U) telophase II-like stage. Green and red arrows highlight FISH signals corresponding to an arm region of chromosome I (BAC F1N21, green) and a subtelomeric region of chromosome II (BAC F11L15, red), respectively (panels $\mathrm{B}, \mathrm{C}, \mathrm{H}, \mathrm{M}-\mathrm{Q}, \mathrm{S}$ and $\mathrm{U}$ ). White arrows highlight chromosome bridges seen during Atcom1-1 meiosis (R, U). Meiotic progression in pollen mother cells was followed after chromosomes were stained with DAPI. Scale bar: $10 \mu \mathrm{m}$.

of the single strand by the recombinase Rad51 for subsequent strand invasion and D-loop formation (Li et al, 2004; Shinohara and Shinohara, 2004). Filament formation can be observed cytologically, as the formation of Rad51 foci upon indirect immunofluorescence (IF) staining. While large numbers of Rad51 foci appear transiently in wild-type prophase cells, only very few (comparable to those found in Atspo11-1-1 and Atmre11-3) are seen in Atcom1-1 cells (Figure 5; Supplementary Figure 4). One interpretation of this result could be that meiotic DSBs are reduced or absent. We therefore performed IF for $\gamma \mathrm{H} 2 \mathrm{AX}$ (SanchezMoran et al, 2007), a phospho form of a histone H2A variant representing a specific, local and fast response to DSBs (Rogakou et al, 1998; Fernandez-Capetillo et al, 2003; Friesner et al, 2005; Supplementary Figure 5B). This control confirmed the presence of high levels of DSBs in Atcom1-1 cells (and in Atmre11-3 and AtRad50 mutant cells), similar to the staining observed in early stages in wild-type meiocytes and consistent with the observation of fragmentation in anaphase I (Figure 3Q-S). Thus, we infer that Atcom1-1 mutants generate DSBs, but do not form Rad51 filaments efficiently.

A critical early step in repair is the removal of Spo11, which remains covalently linked after it has cleaved a step known to depend on Rad50 (; Alani et al, 1990), Mre11 (Nairz and
Klein, 1997; Tsubouchi and Ogawa, 1998) and Com1/Sae2 (McKee and Kleckner, 1997; Prinz et al, 1997) in S. cerevisiae. In such a situation, Spo11 foci were shown to accumulate in mutant nuclei by visualising an epitope-tagged Spo11 (Prieler et al, 2005). We tried to address this question in A. thaliana using an AtSPO11-1-specific antibody (Sanchez-Moran et al, 2007). While AtSPO11-1 was virtually undetectable in meiocytes of wild-type cells $(99.7 \%$ of prophase I cells show no AtSPO11-1 foci, $n=440$ ), the same antibody detected AtSPO11-1 in $98 \%$ of Atcom 1-1 meiocytes $(n=50)$, some of which showed striking AtSPO11-1 hyperaccumulation (30/50 cell showed very intensive staining, 19/50 showed staining with lower intensity) (Figure 5; Supplementary Figure 5A). Interestingly, we find a similar hyperaccumulation of AtSPO11-1 in meiocytes of Atmre11-3 and Atrad50 mutants (Supplementary Figure 5A). We summarise that available evidence places the defect of Atcom1-1 before AtRAD51 filament formation and suggests a problem with AtSPO11-1 removal. Thus, our study suggests that yeast Com1/Sae2 and Arabidopsis AtCOM1 are required for equivalent mechanisms during meiotic DSB repair.

\section{AtCOM1 is required for resistance to mitomycin C}

Aside from meiosis, the $S$. cerevisiae COM1/SAE2 gene is required for genomic stability and for resistance to DNA 


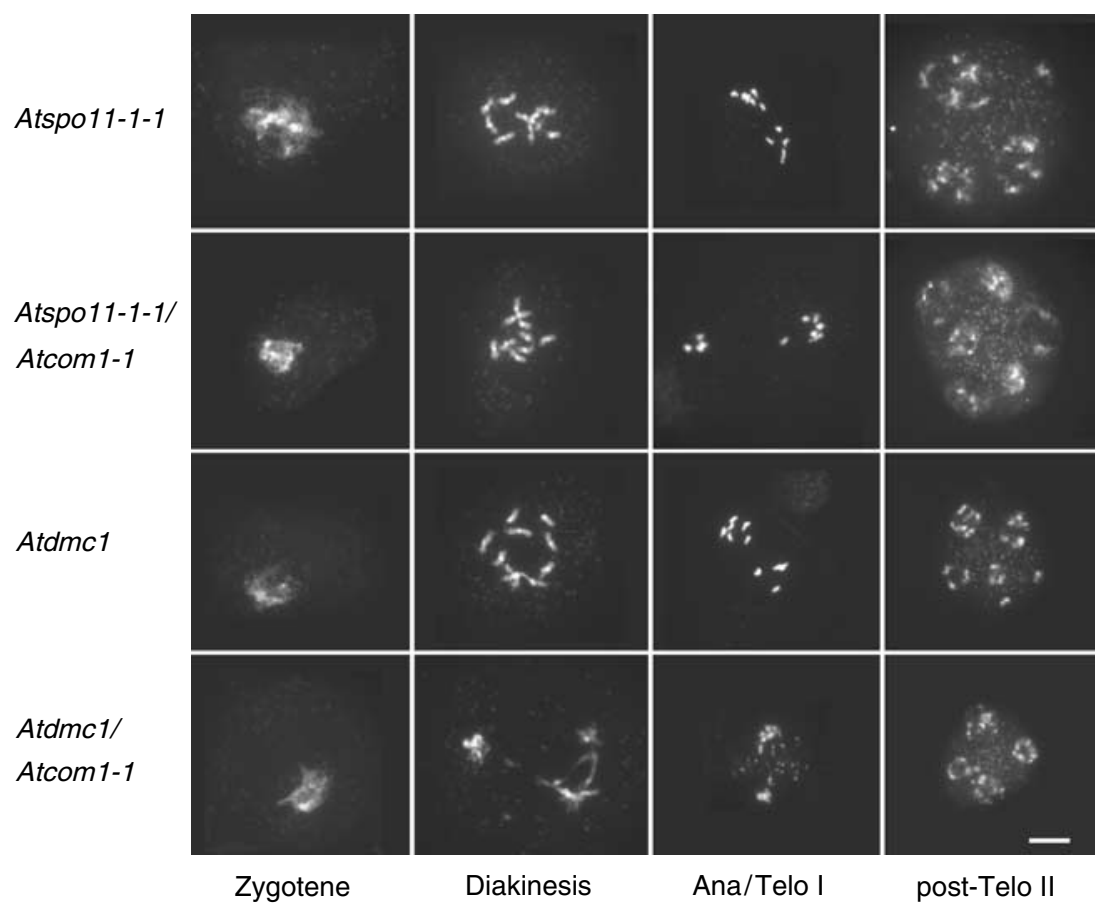

Figure 4 AtCOM1 acts downstream of AtSPO11-1 and upstream of AtDMC1. Comparison of meiotic progression in the Atspo11-1-1 mutant (upper panel) and in the Atcom1-1Atspo11-1-1 double mutant (second panel from top). In both, zygotene-like stages are followed by progressive condensation and formation of univalents, which subsequently segregate at random, forming polyads at the end of meiosis II. Comparison of meiotic progression in the Atdmc1 mutant (third panel from top) and in the Atcom1-1Atdmc1 double mutant (lowest panel). Whereas Atdmc1 mutants form univalents, which segregate at random to give rise to polyads at the end of meiosis II, the Atcom 1-1Atdmc1 double mutant resembles Atcom1-1, with fragmented chromosomes. Chromosomes are stained with DAPI. Scale bar: $10 \mu \mathrm{m}$.

A

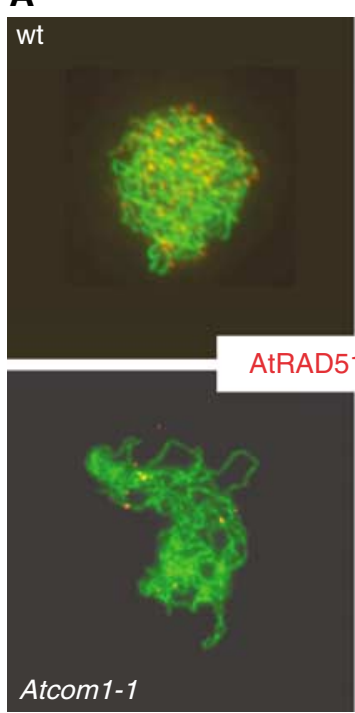

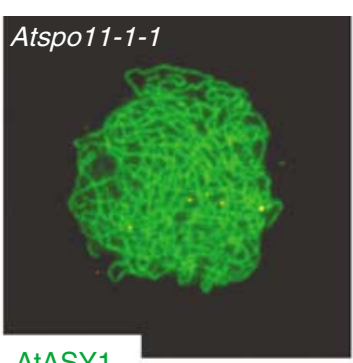

AtASY1

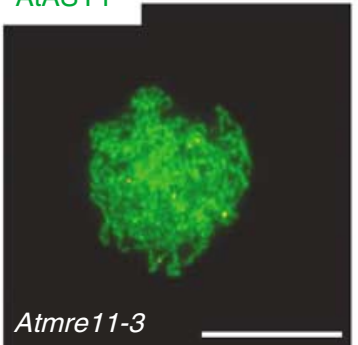

B

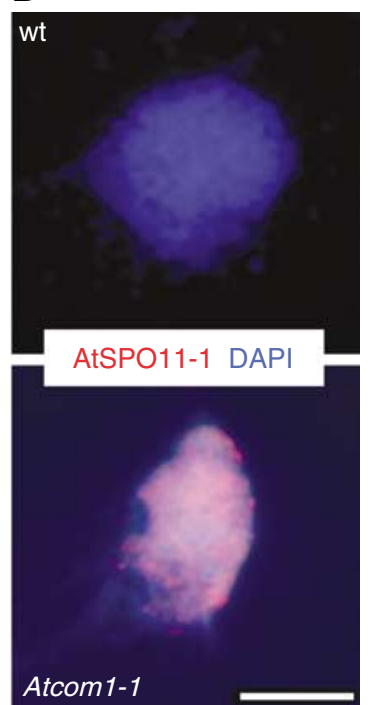

Figure 5 Atcom1-1 cells fail to form AtRAD51 foci, but accumulate AtSPO11-1. (A) Atcom1-1 cells fail to form AtRAD51 foci. Immunofluorescent staining of spreads of meiotic cells with antibodies directed against the axial element protein Asy1 (green) and against AtRad51 (red). Whereas in wild type (wt) numerous AtRAD51 foci are observed in zygotene, representing loci of meiotic DNA repair and recombination, only very few foci are seen in Atspo11-1-1, Atcom1-1 and Atmre11-3 mutants. Scale bar: $10 \mu$ m. (B) AtSPO11-1 is enriched in prophase I of Atcom1-1 mutant meiosis. Immunofluorescent staining of spreads of cells in prophase I stages of wild type (wt) and Atcom1-1 meiocytes with an antibody directed against AtSPO11-1 (red). DNA was counterstained with DAPI (blue). Scale bar: $10 \mu \mathrm{m}$.

cross-linking agents, including mitomycin $\mathrm{C}(\mathrm{MMC})$ in vegetative cells (Lobachev et al, 2002; Wu et al, 2004). Figure 2B shows wild-type and Atcom1-1 seedlings, which were germinated on media containing $40 \mu \mathrm{M}$ MMC. In contrast to wild type, true leaves do not develop in the mutant, while expansion of cotyledons, which does not require cell division, is unaffected. The strong MMC sensitivity was $100 \%$ penetrant for homozygous Atcom1-1 mutants, whereas heterozygous or 
wild-type plants were resistant. However, no difference in sensitivity to a number of other genotoxic agents was found in a series of experiments described in the Supplementary data. In addition, in yeast, diploid com $1 / \operatorname{sae} 2 \Delta$ mutants were only very weakly sensitive to X-ray or a radiomimetic alkylating agent in our hands (McKee and Kleckner, 1997; Prinz et al, 1997). Work in recent years has implicated Com $1 /$ Sae2 in the processing of very selective lesions such as hairpin capped DNA ends (data not shown; Rattray et al, 2001; Lobachev et al, 2002) or interstrand cross-links (Wu et al, 2004). The selective sensitivity of vegetative cells of both Atcom 1-1 and yeast com $1 /$ sae $2 \Delta$ mutants to MMC is further evidence for the conservation of their roles in evolution and, furthermore, may reflect a specificity of AtCOM1 for the repair of only a subset of DNA lesions.

\section{Discussion}

This study describes the first identification and characterisation of a Com1/Sae2 homologue in a higher eukaryote, the AtCOM1 gene of A. thaliana. This study provides evidence that both female and male meiosis are affected by the Atcom 1 mutation, which thus leads to sterility. Atcom1 mutants do not form any viable microspores or macrospores, as judged from our analysis of gametogenesis. Furthermore, Atcom 1 mutants do not form seeds, even when pollinated with wild-type pollen. Analysis of DAPI-stained chromosomes reveals that during progression of both male and female meiosis, severe chromosome fragmentation occurs. Chromosome pairing, visualised by DAPI and FISH analysis, never occurred in Atcom 1 mutants. Chromosome fragmentation and failure of pairing indicate that meiotic DSBs are produced, but downstream events such as DNA end processing or heteroduplex formation are impaired in Atcom 1 mutants. Epistasis analysis clearly shows that the DNA fragmentation observed in Atcom 1 mutant plants depends on the activity of the AtSPO11-1 protein. This result places Atcom1 downstream of Atspo11-1. In contrast, Atcom1-1 Atdmc1 double mutants show severe chromosome fragmentation and, therefore, resemble the Atcom1-1 mutant rather than the Atdmc1 mutant. This result implies that meiotic DSBs are formed in Atdmc1 mutants, and that their repair depends on AtCOM1. This result places AtCOM1 upstream of the RecA-related DMC1 protein.

The mechanistic role of Com1/Sae2 is still unknown. Nevertheless, it has been established that Com1/Sae2 is needed for the removal of Spo11 from DNA during meiosis in yeast. As an intermediate of the DNA cleavage reaction, Spo11 is covalently bound to the $5^{\prime}$ end phosphate of the DNA at the DSB site. Removal of the Spoll protein occurs by nicking the DNA next to the cleavage site, thereby releasing the catalytic protein attached to a few nucleotides (Neale et al, 2005). If Com $1 / \mathrm{Sae} 2$ is not present, this reaction does not occur and subsequent steps in meiotic progression are blocked. The present study reveals that the plant protein AtCOM1 is involved at the same stage during meiosis. Moreover, evidence is presented that Atcom 1-1 mutant plants accumulate the AtSpo11-1 protein during prophase I of meiosis. Furthermore, the strand invasion mediator AtRAD51, which forms numerous foci on meiotic chromosomes and is visible from leptotene to pachytene in wild-type meiocytes ( $\mathrm{Li}$ et al, 2004), cannot be detected in Atcom-1-1 mutants. These results imply that processing of DSBs is impaired in mutant plants because of AtSPO11-1 not being removed from DNA ends at DSB sites, thereby blocking processing of DNA ends. Future experiments are needed to resolve the question as to whether the second, meiotically active Arabidopsis SPO11 protein, AtSPO11-2, behaves similarly to AtSPO11-1 in an Atcom 1 mutant background.

\section{Somatic function of the AtCOM1 protein}

Expression data for the AtCOM1 gene, presented by Deveaux et al (2000) and confirmed by this study, showed strong accumulation of the AtCOM1 transcript upon exposure to IR in somatic tissues. Furthermore, data presented by Garcia et al (2003) show that transcription of AtCOM1 is strictly dependent on ATM, a conserved protein kinase known to mediate signalling of DNA lesions (Shiloh, 1998). Thus, to unravel a potential somatic function of the AtCOM1 protein, the Atcom1-1 mutant was tested for resistance to various genotoxic treatments. Under the conditions tested, only the presence of MMC led to strong inhibition of mutant seedling growth. In contrast, all other treatments including IR, did not affect the growth of homozygous Atcom1-1 mutants compared to heterozygous or wild-type plants. This may reflect a specificity of AtCOM1's involvement in the repair of a subset of DNA lesions. It is conceivable that, upon DNA damage, ATM-mediated signalling leads to broad transcriptional induction of DNA repair-related genes, and that it depends on the actual type of DNA damage, which of the corresponding gene products is effectively needed for repair. In somatic tissues, AtCOM1 seems to be needed for the repair of interstrand DNA cross-links, the dominant effect of MMC, but not for other types of DNA damage, which are predominantly formed by IR, HU, cisplatin, bleomycin or aphidicolin. The selective sensitivity of vegetative cells of both Atcom1-1 and yeast com $1 /$ sae $2 \Delta$ mutants to DNA crosslinking agents provides further evidence for the conservation of their roles in evolution.

\section{COM1/SAE2 homologues are present in higher eukaryotes}

As demonstrated in Figure 1B, AtCOM1 is much more closely related to the vertebrate COM1/SAE2 homologues than to the fungal branch. The mammalian relative of AtCOM1 and, thus, also of yeast Com1/Sae2, is CtIP (Fusco et al, 1998; Schaeper et al, 1998), which has been strongly implicated in DNA repair. Initially, CtIP was identified as a protein that binds to Rb, BRCA1 and CtBP1 (Fusco et al, 1998; Schaeper et al, 1998; Meloni et al, 1999; Yu and Baer, 2000). CtIP's interaction with the transcriptional regulators $\mathrm{CtBP}, \mathrm{Rb}$ and LMO4 involves it in the regulaton of cell-cycle and DNA repair-related proteins (Yu et al, 1998; Sum et al, 2002; Liu and Lee, 2006). CtIP is most highly expressed in thymus and testis (Wong et al, 1998), where DNA DSBs are naturally generated. Yu et al (2006) show that CtIP is ubiquitinated in a BRCA1/BARD1-dependent manner following DNA damage and that this modification leads to its association with chromatin, not to its degradation. Upon DNA damage, CtIP associates with BRCA1/BARD1, Rad50, Mre11 and NBS1 (Greenberg et al, 2006). Furthermore, CtIP's interaction with BRCA1 seems to be essential for the activation of a DNA damage checkpoint in G2 (Yu and Chen, 2004; Greenberg et al, 2006), possibly mediated by CtIP's phosphorylation by ATM, a process that depends on BRCA1 (Foray et al, 2003). Homozygous Ctip $^{-/-}$-knockout mice die during 
embryonic stage 4 and heterozygous Ctip ${ }^{+/-}$mice are prone to developing tumours (Chen et al, 2005). While all previous data are consistent with a role of CtIP in DSB repair similar to Com1/Sae2, these observations have not provided a clue regarding the molecular role of CtIP. Both CtIP and Com1/ Sae2 are phosphorylated by ATM/Tel1 kinases in response to DNA damage (Foray et al, 2003; Baroni et al, 2004), cooperate with Mre11 and Rad50 (Lobachev et al, 2002; Lisby et al, 2004; Greenberg et al, 2006) and play a role in DNA damage checkpoint signalling (Yu and Chen, 2004; Clerici et al, 2006). CtIP, however, also interacts physically with BRCA1, Rb and CtBP1, which are not part of the yeast DNA repair repertoire. Thus, CtIP and possibly AtCOM1 may have acquired accessory functions in addition to those conserved with yeast Com1/Sae2. So far, a detailed analysis of CtIP function has been complicated by the embryonic lethality caused by homozygous CtIP deficiency in mice. However, heterozygous CtIP leads to increased incidence of cancer (Chen et al, 2005). Our findings lead to the prediction that CtIP is required for meiotic DSB repair and for resistance to certain genotoxic agents (e.g. MMC). CtIP may function by modulating the nuclease activity of Mre11 and may be an important factor in processing hairpin capped DNA ends and DNA ends blocked by covalently bound proteins (such as in topoisomerase II in the presence of topo II poisons). Interestingly, deletion of RAD50 is embryonic-lethal (Luo et al, 1999) and a hypomorphic mutation of MRE11 severely compromises embryo viability (Theunissen et al, 2003) in mammals, just as deletion of CtIP (Chen et al, 2005), but all three genes are not essential in yeast and in plants. This is consistent with evolutionary conservation of a potential regulatory role of Com1/Sae2 for the Mre11 complex. The function of yeast Com1/Sae2 may overlap with that of flap endonuclease Fen1, because they display synthetic lethality, a relation that might also be conserved between mammalian Fen1 and CtIP. Mammalian Fen1 suppresses tumour progression (Kucherlapati et al, 2002). The phenotype of Atcom 1-1 provides information which links yeast Com1/Sae2 and mammalian CtIP. Based on the detailed knowledge on Com1/Sae2, we suggest CtIP to be required for genome stability as a tumour suppressor, for fertility through meiotic DNA repair and possibly for $\mathrm{V}$ (D) J recombination, because in yeast Com1/Sae2 is instrumental together with Mre11 to process hairpins (Rattray et al, 2001; Lobachev et al, 2002 our own observations, manuscript in preparation). Com1/Sae2 and CtIP have been studied separately, but the knowledge of their relationship should strongly stimulate both hitherto separated fields.

\section{Materials and methods}

\section{Plant growth conditions and plant transformation}

All plants were germinated on soil after 2 days of stratification in water at $4^{\circ} \mathrm{C}$. Plants were grown at $22^{\circ} \mathrm{C}$ with a $16: 8 \mathrm{~h}$ light: dark photoperiod. Seeds of the SALK_061706 (Atcom1-1) and SALK_122386 (Atcom1-2) lines were obtained from the Nottingham Arabidopsis Stock Center (Nottingham, UK). Mutants and double mutants were identified by PCR (see Supplementary data). Transformation of plants heterozygous for the Atcom 1-1 mutation with pBIB-Hyg/gAtCOM1, utilising Agrobacterium tumefaciens strain GV3101, was performed as described previously (Bechtold et al, 1993).

\section{Vector construction}

For construction of the complementing pBIB-Hyg/gAtCOM1, a 4811 bp genomic DNA fragment derived from BAC clone ATF4F15 (ABRC, Columbus, Ohio) by digestion with BsmI and subsequent T4 polymerase treatment, was cloned into the SmaI restrictions site of the plant binary vector pBIB-Hyg (Becker, 1990) and subsequently used for transformation.

\section{Analysis of meiotic chromosomes}

DAPI staining of male meiotic chromosomes was performed as described previously (Ross et al, 1996). Female meiotic chromosomes were observed as described previously (Motamayor et al, 2000), except that pictures were taken with a Zeiss Axioplan 2 microscope, including an LSM 510 Laser module. Data processing was performed with Zeiss LSM 510 Meta and Helicon Focus software. FISH and fluorescent immunolocalisation analysis of ASY1 and AtRAD51 was performed as described previously (Kerzendorfer et al, 2006), except that both the anti-RAD51 antibody (rat) and the anti-ASY1 antibody (rabbit) were used in 1:500 dilutions. The polyclonal rabbit antibody against AtSPO11-1 (a gift from F.C.H Franklin) was diluted 1:100 and a polyclonal rabbit anti-phospho-H2AX (Ser 139) (Upstate) antibody was diluted 1:100. Immunolocalisation analysis of AtSPO11-1 and $\gamma \mathrm{H} 2 \mathrm{AX}$ antibodies was carried out on squashed prophase I meiocytes from Atcom 1-1 mutant plants as described previously (Page et al, 1998; Sanchez-Moran et al, 2007).

\section{Yeast spore viability assay}

Yeast strain SK1 (with the following relevant genotype: MATa/ alpha, ura3/ura3, com1::KanMX4/com 1::KanMX4), was transformed with plasmid pRS316, including the COM1/SAE2 coding sequence (with additional $682 \mathrm{bp}$ upstream and $251 \mathrm{bp}$ downstream regions of the COM1/SAE2 coding sequence) between the SacII and HpaI restriction sites. Indicated point mutations were introduced by standard techniques and verified by sequencing. Yeast spore viability was determined as described by Prinz and co-workers (Prinz et al, 1997).

\section{Supplementary data}

Supplementary data are available at The EMBO Journal Online (http://www.embojournal.org)

\section{Acknowledgements}

We thank Dieter Schweizer, Josef Loidl, Verena Jantsch and Alexandra Penkner for critical reading of the manuscript and/or stimulating discussions. We are grateful to Chris Franklin for sharing antibodies directed against ASY1, AtRAD51 and AtSPO111. We thank René Ladurner for help with FISH analysis, MarieTherese Kurzbauer for supporting the AtCOM1 expression analysis and Fritz Hunger for plant genotyping. PS is supported by an APART fellowship of the Austrian Academy of Sciences. This work was supported by a grant from the Austrian Science Foundation FWF (P19307) to PS.

\section{References}

Akutsu N, Iijima K, Hinata T, Tauchi H (2007) Characterization of the plant homolog of Nijmegen breakage syndrome 1: involvement in DNA repair and recombination. Biochem Biophys Res Commun 353: 394-398
Alani E, Padmore R, Kleckner N (1990) Analysis of wild-type and rad50 mutants of yeast suggests an intimate relationship between meiotic chromosome synapsis and recombination. Cell 61: $419-436$ 
Alexander MP (1969) Differential staining of aborted and nonaborted pollen. Stain Technol 44: 117-122

Alonso JM, Stepanova AN, Leisse TJ, Kim CJ, Chen H, Shinn P, Stevenson DK, Zimmerman J, Barajas P, Cheuk R, Gadrinab C, Heller C, Jeske A, Koesema E, Meyers CC, Parker H, Prednis L, Ansari Y, Choy N, Deen H et al (2003) Genome-wide insertional mutagenesis of Arabidopsis thaliana. Science 301: 653-657

Altschul SF, Madden TL, Schaffer AA, Zhang J, Zhang Z, Miller W, Lipman DJ (1997) Gapped BLAST and PSI-BLAST: a new generation of protein database search programs. Nucleic Acids Res 25: 3389-3402

Baroni E, Viscardi V, Cartagena-Lirola H, Lucchini G, Longhese MP (2004) The functions of budding yeast Sae2 in the DNA damage response require Mec1- and Tel1-dependent phosphorylation. Mol Cell Biol 24: 4151-4165

Bechtold N, Ellis J, Pelletier G (1993) In planta Agrobacterium mediated gene transfer by infiltration of adult Arabidopsis thaliana plants. C R Acad Sci Paris 316: 1194-1199

Becker D (1990) Binary vectors which allow the exchange of plant selectable markers and reporter genes. Nucleic Acids Res 18: 203

Bergerat A, de Massy B, Gadelle D, Varoutas PC, Nicolas A, Forterre P (1997) An atypical topoisomerase II from Archaea with implications for meiotic recombination. Nature 386: 414-417

Bhatt AM, Canales C, Dickinson HG (2001) Plant meiosis: the means to $1 \mathrm{~N}$. Trends Plant Sci 6: 114-121

Birrell GW, Brown JA, Wu HI, Giaever G, Chu AM, Davis RW, Brown JM (2002) Transcriptional response of Saccharomyces cerevisiae to DNA-damaging agents does not identify the genes that protect against these agents. Proc Natl Acad Sci USA 99: 8778-8783

Bleuyard JY, Gallego ME, White CI (2004) Meiotic defects in the Arabidopsis rad50 mutant point to conservation of the MRX complex function in early stages of meiotic recombination. Chromosoma 113: 197-203

Bundock P, Hooykaas P (2002) Severe developmental defects, hypersensitivity to DNA-damaging agents, and lengthened telomeres in Arabidopsis MRE11 mutants. Plant Cell 14: 2451-2462

Cartagena-Lirola H, Guerini I, Viscardi V, Lucchini G, Longhese MP (2006) Budding Yeast Sae2 is an in vivo target of the Mec1 and Tel1 checkpoint kinases during meiosis. Cell Cycle 5: 1549-1559

Chen PL, Liu F, Cai S, Lin X, Li A, Chen Y, Gu B, Lee EY, Lee WH (2005) Inactivation of CtIP leads to early embryonic lethality mediated by G1 restraint and to tumorigenesis by haploid insufficiency. Mol Cell Biol 25: 3535-3542

Clerici M, Mantiero D, Lucchini G, Longhese MP (2005) The Saccharomyces cerevisiae Sae2 protein promotes resection and bridging of double strand break ends. J Biol Chem 280: 38631-38638

Clerici M, Mantiero D, Lucchini G, Longhese MP (2006) The Saccharomyces cerevisiae Sae2 protein negatively regulates DNA damage checkpoint signalling. EMBO Rep 7: 212-218

Couteau F, Belzile F, Horlow C, Grandjean O, Vezon D, Doutriaux MP (1999) Random chromosome segregation without meiotic arrest in both male and female meiocytes of a dmc1 mutant of Arabidopsis. Plant Cell 11: 1623-1634

D'Amours D, Jackson SP (2002) The Mre11 complex: at the crossroads of dna repair and checkpoint signalling. Nat Rev Mol Cell Biol 3: 317-327

Daoudal-Cotterell S, Gallego ME, White CI (2002) The plant Rad50Mre11 protein complex. FEBS Lett 516: 164-166

Deng C, Brown JA, You D, Brown JM (2005) Multiple endonucleases function to repair covalent topoisomerase I complexes in Saccharomyces cerevisiae. Genetics 170: 591-600

Deveaux Y, Alonso B, Pierrugues O, Godon C, Kazmaier M (2000) Molecular cloning and developmental expression of AtGR1, a new growth-related Arabidopsis gene strongly induced by ionizing radiation. Radiat Res 154: 355-364

Edgar RC (2004) MUSCLE: multiple sequence alignment with high accuracy and high throughput. Nucleic Acids Res 32: 1792-1797

Fernandez-Capetillo O, Mahadevaiah SK, Celeste A, Romanienko PJ, Camerini-Otero RD, Bonner WM, Manova K, Burgoyne P, Nussenzweig A (2003) H2AX is required for chromatin remodeling and inactivation of sex chromosomes in male mouse meiosis. Dev Cell 4: 497-508

Foray N, Marot D, Gabriel A, Randrianarison V, Carr AM, Perricaudet M, Ashworth A, Jeggo P (2003) A subset of ATMand ATR-dependent phosphorylation events requires the BRCA1 protein. EMBO J 22: 2860-2871
Frickey T, Lupas A (2004) CLANS: a Java application for visualizing protein families based on pairwise similarity. Bioinformatics 20: 3702-3704

Friesner JD, Liu B, Culligan K, Britt AB (2005) Ionizing radiationdependent gamma-H2AX focus formation requires ataxia telangiectasia mutated and ataxia telangiectasia mutated and Rad3related. Mol Biol Cell 16: 2566-2576

Fusco C, Reymond A, Zervos AS (1998) Molecular cloning and characterization of a novel retinoblastoma-binding protein. Genomics 51: 351-358

Gallego ME, Jeanneau M, Granier F, Bouchez D, Bechtold N, White CI (2001) Disruption of the Arabidopsis RAD50 gene leads to plant sterility and MMS sensitivity. Plant $J$ 25: 31-41

Garcia V, Bruchet H, Camescasse D, Granier F, Bouchez D, Tissier A (2003) AtATM is essential for meiosis and the somatic response to DNA damage in plants. Plant Cell 15: 119-132

Greenberg RA, Sobhian B, Pathania S, Cantor SB, Nakatani Y, Livingston DM (2006) Multifactorial contributions to an acute DNA damage response by BRCA1/BARD1-containing complexes. Genes Dev 20: 34-46

Grelon M, Vezon D, Gendrot G, Pelletier G (2001) AtSPO11-1 is necessary for efficient meiotic recombination in plants. EMBO J 20: $589-600$

Hartung F, Puchta H (2000) Molecular characterisation of two paralogous SPO11 homologues in Arabidopsis thaliana. Nucleic Acids Res 28: 1548-1554

Hartung F, Puchta H (2001) Molecular characterization of homologues of both subunits A (SPO11) and B of the archaebacterial topoisomerase 6 in plants. Gene 271: 81-86

Hunter N, Kleckner N (2001) The single-end invasion: an asymmetric intermediate at the double-strand break to doubleholliday junction transition of meiotic recombination. Cell 106: $59-70$

Keeney S, Giroux CN, Kleckner N (1997) Meiosis-specific DNA double-strand breaks are catalyzed by Spo11, a member of a widely conserved protein family. Cell 88: 375-384

Keeney S, Kleckner N (1995) Covalent protein-DNA complexes at the $5^{\prime}$ strand termini of meiosis-specific double-strand breaks in yeast. Proc Natl Acad Sci USA 92: 11274-11278

Keeney S (2001) Mechanism and control of meiotic recombination initiation. Curr Top Dev Biol 52: 1-53

Kerzendorfer C, Vignard J, Pedrosa-Harand A, Siwiec T, Akimcheva S, Jolivet S, Sablowski R, Armstrong S, Schweizer D, Mercier R, Schlogelhofer P (2006) The Arabidopsis thaliana MND1 homologue plays a key role in meiotic homologous pairing, synapsis and recombination. J Cell Sci 119: 2486-2496

Kucherlapati M, Yang K, Kuraguchi M, Zhao J, Lia M, Heyer J, Kane MF, Fan K, Russell R, Brown AM, Kneitz B, Edelmann W, Kolodner RD, Lipkin M, Kucherlapati R (2002) Haploinsufficiency of Flap endonuclease (Fen1) leads to rapid tumor progression. Proc Natl Acad Sci USA 99: 9924-9929

Li W, Chen C, Markmann-Mulisch U, Timofejeva L, Schmelzer E, Ma H, Reiss B (2004) The Arabidopsis AtRAD51 gene is dispensable for vegetative development but required for meiosis. Proc Natl Acad Sci USA 101: 10596-10601

Lisby M, Barlow JH, Burgess RC, Rothstein R (2004) Choreography of the DNA damage response: spatiotemporal relationships among checkpoint and repair proteins. Cell 118: 699-713

Liu F, Lee WH (2006) CtIP activates its own and cyclin D1 promoters via the E2F/RB pathway during G1/S progression. Mol Cell Biol 26: $3124-3134$

Lobachev KS, Gordenin DA, Resnick MA (2002) The Mrell complex is required for repair of hairpin-capped double-strand breaks and prevention of chromosome rearrangements. Cell 108: $183-193$

Luo G, Yao MS, Bender CF, Mills M, Bladl AR, Bradley A, Petrini JH (1999) Disruption of mRad50 causes embryonic stem cell lethality, abnormal embryonic development, and sensitivity to ionizing radiation. Proc Natl Acad Sci USA 96: 7376-7381

Marchler-Bauer A, Panchenko AR, Ariel N, Bryant SH (2002) Comparison of sequence and structure alignments for protein domains. Proteins 48: 439-446

McKee AH, Kleckner N (1997) A general method for identifying recessive diploid-specific mutations in Saccharomyces cerevisiae, its application to the isolation of mutants blocked at intermediate stages of meiotic prophase and characterization of a new gene SAE2. Genetics 146: 797-816 
Meloni AR, Smith EJ, Nevins JR (1999) A mechanism for Rb/p130mediated transcription repression involving recruitment of the CtBP corepressor. Proc Natl Acad Sci USA 96: 9574-9579

Motamayor JC, Vezon D, Bajon C, Sauvanet A, Grandjean O, Marchand M, Bechtold N, Pelletier G, Horlow C (2000) Switch (swi1), an Arabidopsis thaliana mutant affected in the female meiotic switch. Sex Plant Reprod 12: 209-218

Nairz K, Klein F (1997) mre11S-a yeast mutation that blocks double-strand-break processing and permits nonhomologous synapsis in meiosis. Genes Dev 11: 2272-2290

Neale MJ, Keeney S (2006) Clarifying the mechanics of DNA strand exchange in meiotic recombination. Nature 442: 153-158

Neale MJ, Pan J, Keeney S (2005) Endonucleolytic processing of covalent protein-linked DNA double-strand breaks. Nature 436: 1053-1057

Page J, Suja JA, Santos JL, Rufas JS (1998) Squash procedure for protein immunolocalization in meiotic cells. Chromosome Res $\mathbf{6}$ : 639-642

Page SL, Hawley RS (2003) Chromosome choreography: the meiotic ballet. Science 301: 785-789

Paques F, Haber JE (1999) Multiple pathways of recombination induced by double-strand breaks in Saccharomyces cerevisiae. Microbiol Mol Biol Rev 63: 349-404

Prieler S, Penkner A, Borde V, Klein F (2005) The control of Spo11's interaction with meiotic recombination hotspots. Genes Dev 19: 255-269

Prinz S, Amon A, Klein F (1997) Isolation of COM1, a new gene required to complete meiotic double-strand break-induced recombination in Saccharomyces cerevisiae. Genetics 146: 781-795

Puizina J, Siroky J, Mokros P, Schweizer D, Riha K (2004) Mre11 deficiency in Arabidopsis is associated with chromosomal instability in somatic cells and Spo11-dependent genome fragmentation during meiosis. Plant Cell 16: 1968-1978

Rattray AJ, McGill CB, Shafer BK, Strathern JN (2001) Fidelity of mitotic double-strand-break repair in Saccharomyces cerevisiae: a role for SAE2/COM1. Genetics 158: 109-122

Rice P, Longden I, Bleasby A (2000) EMBOSS: the European Molecular Biology Open Software Suite. Trends Genet 16: 276-277

Rogakou EP, Pilch DR, Orr AH, Ivanova VS, Bonner WM (1998) DNA double-stranded breaks induce histone H2AX phosphorylation on serine 139. J Biol Chem 273: 5858-5868

Ross KJ, Fransz P, Jones GH (1996) A light microscopic atlas of meiosis in Arabidopsis thaliana. Chromosome Res 4: $507-516$

Sanchez-Moran E, Santos JL, Jones GH, Franklin FC (2007) ASY1 mediates AtDMC1-dependent interhomolog recombination during meiosis in Arabidopsis. Genes Dev 21: 2220-2233

Schaeper U, Subramanian T, Lim L, Boyd JM, Chinnadurai G (1998) Interaction between a cellular protein that binds to the C-terminal region of adenovirus E1A (CtBP) and a novel cellular protein is disrupted by E1A through a conserved PLDLS motif. J Biol Chem 273: $8549-8552$
Schwacha A, Kleckner N (1997) Interhomolog bias during meiotic recombination: meiotic functions promote a highly differentiated interhomolog-only pathway. Cell 90: 1123-1135

Shiloh Y (1998) Ataxia-telangiectasia, ATM and genomic stability: maintaining a delicate balance. Two international workshops on ataxia-telangiectasia, related disorders and the ATM protein. Biochim Biophys Acta 1378: R11-R18

Shinohara A, Shinohara M (2004) Roles of RecA homologues Rad51 and Dmc1 during meiotic recombination. Cytogenet Genome Res 107: $201-207$

Smith KN, Nicolas A (1998) Recombination at work for meiosis. Curr Opin Genet Dev 8: 200-211

Stacey NJ, Kuromori T, Azumi Y, Roberts G, Breuer C, Wada T, Maxwell A, Roberts K, Sugimoto-Shirasu K (2006) Arabidopsis SPO11-2 functions with SPO11-1 in meiotic recombination. Plant $J$ 48: 206-216

Sum EY, Peng B, Yu X, Chen J, Byrne J, Lindeman GJ, Visvader JE (2002) The LIM domain protein LMO4 interacts with the cofactor CtIP and the tumor suppressor BRCA1 and inhibits BRCA1 activity. J Biol Chem 277: 7849-7856

Theunissen JW, Kaplan MI, Hunt PA, Williams BR, Ferguson DO, Alt FW, Petrini JH (2003) Checkpoint failure and chromosomal instability without lymphomagenesis in Mre11(ATLD1/ATLD1) mice. Mol Cell 12: 1511-1523

Tsubouchi H, Ogawa H (1998) A novel mrell mutation impairs processing of double-strand breaks of DNA during both mitosis and meiosis. Mol Cell Biol 18: 260-268

Wong AK, Ormonde PA, Pero R, Chen Y, Lian L, Salada G, Berry S, Lawrence Q, Dayananth P, Ha P, Tavtigian SV, Teng DH, Bartel PL (1998) Characterization of a carboxy-terminal BRCA1 interacting protein. Oncogene 17: 2279-2285

Wu HI, Brown JA, Dorie MJ, Lazzeroni L, Brown JM (2004) Genome-wide identification of genes conferring resistance to the anticancer agents cisplatin, oxaliplatin, and mitomycin C. Cancer Res 64: 3940-3948

Yin Y, Cheong H, Friedrichsen D, Zhao Y, Hu J, Mora-Garcia S, Chory J (2002) A crucial role for the putative Arabidopsis topoisomerase VI in plant growth and development. Proc Natl Acad Sci USA 99: 10191-10196

Yu X, Baer R (2000) Nuclear localization and cell cycle-specific expression of CtIP, a protein that associates with the BRCA1 tumor suppressor. J Biol Chem 275: 18541-18549

Yu X, Chen J (2004) DNA damage-induced cell cycle checkpoint control requires CtIP, a phosphorylation-dependent binding partner of BRCA1 C-terminal domains. Mol Cell Biol 24: 9478-9486

Yu X, Fu S, Lai M, Baer R, Chen J (2006) BRCA1 ubiquitinates its phosphorylation-dependent binding partner CtIP. Genes Dev 20: $1721-1726$

Yu X, Wu LC, Bowcock AM, Aronheim A, Baer R (1998) The C-terminal (BRCT) domains of BRCA1 interact in vivo with CtIP, a protein implicated in the CtBP pathway of transcriptional repression. J Biol Chem 273: 25388-25392

Zickler D, Kleckner N (1999) Meiotic chromosomes: integrating structure and function. Annu Rev Genet 33: 603-754 